\title{
ORIENTED RELATIVE EMBEDDINGS OF GRAPHS
}

0. It is generally known that, when a connected graph $G$ is a 2-cell embedded on a closed surface, any face of the embedding is bounded by a closed walk in $G$. An interesting question is which closed walks appear as the boundaries of embeddings. More exactly, given a set of (oriented) closed walks $W$ in $G$, is there a 2-cell embedding of $G$ on some surface realizing $W$, i.e., such that each of the prescribed walks bounds a face of the embedding? If so, such an embedding will be called a relative embedding of $G$ with respect to $W$.

Similar questions have already been studied, even in connection with unexpected applications. Goldstein and Turner [2] dealt, in fact, with relative embeddings of cubic Hamiltonian graphs with respect to a Hamiltonian circuit. Their results are then used to answer some questions from combinatorial group theory. The same class of embeddings was investigated by Marx [6] in the context of analytic functions. Another example is [11] where Vo presents a necessary and sufficient condition for circuits in a planar graph to be realizable in the plane. This is closely related to efficient algorithms constructing planar embeddings. There are also other approaches to realizability. In [3], Section 8, the prescribed walks are not assumed to be closed, and in [7] and [8] the authors study a vertex version of realizability.

In this paper we emphasize the analogy between the relative embeddings and the embeddings in the usual sense. We show that some basic results on relative embeddings, such as Edmonds' combinatorial characterizations or Duke's interpolation theorem, can be obtained by an appropriate modification of known permutation techniques, preserving the spirit of their original proof.

1. Preliminaries. Compact orientable 2-manifolds are referred to as surfaces. Moreover, on each surface a definite orientation is chosen; this makes the surface oriented. We define an oriented 2-cell embedding of a graph as a 2-cell embedding on some oriented surface $X$ in which the faces share 
the same orientation as $X$. Any edge of a graph is provided with an orientation. The term arc will denote an edge with a specific choice of orientation. The symbol $x^{-1}$ denotes the reverse of the $\operatorname{arc} x$. By $E(G)$ and $D(G)$ we denote the sets of edges and arcs of $G$, respectively. For each vertex $v$ of $G$, let $D(v)$ be the set of all arcs at $v$, i.e., emanating from $v$.

This work makes an extensive use of permutations. We write an argument on the right of the permutation. Thus, the composition is to be read from the right to the left. For a permutation $\pi$ the symbol $|\pi|$ denotes the number of disjoint cyclic factors of $\pi$.

Let $G$ be a graph, and $v$ a vertex of $G$. A local rotation at $v$ is a cyclic permutation $P_{v}$ of $D(v)$. A rotation of $G$ is the product

$$
P=\prod_{v} P_{v}
$$

of local rotations at each vertex $v$ of $G$. Thus, $P$ is a permutation on $D(G)$. Let $\omega$ be the permutation of $D(G)$ which assigns $x^{-1}$ to any $\operatorname{arc} x$ of $D(G)$. The product $Q=P \omega$ will be called a corotation of $G$. It is well known how rotations are induced by 2-cell embeddings (see, e.g., [12]). Note that the permutation $Q^{-1}$ corresponds to the face boundaries oriented consistently with the orientation of the surface.

As observed, the boundary of any face of a 2-cell embedding of $G$ is a closed walk in $G$. In the standard terminology, the term "closed walk" is regarded as a certain alternating sequence of vertices and arcs emanating from them. For our purposes it is more convenient to view walks as cyclic permutations, as suggested by the correspondence above. More precisely, we define a walk to be a cyclic permutation $W$ of a subset $D^{\prime}$ of $D(G)$ such that for any $a \in D^{\prime}$ the terminal vertex of $a$ is equal to the initial vertex of $W(a)$. Defining a walk as a cyclic permutation we thereby assigned a fixed orientation to it. Thus, each walk is closed and no its vertex is specified as a base vertex.

A set of walks $W=\left\{W_{1}, \ldots, W_{k}\right\}$ is called admissible if $W_{1}, \ldots, W_{k}$ appear as disjoint cyclic factors of a permutation of $D(G)$. That is, no edge of $G$ it used in $W$ more than once with the same orientation. This allows us to identify the set of admissible walks $W$ with the product $\prod_{i=1}^{k} W_{i}$ (the empty product is the identity permutation).

In our approach to relative embeddings it appears convenient to employ words. A word is an arbitrary (possibly cyclic) sequence of distinct elements (symbols) of $D(G)$. Cyclic words will always be written in parentheses. Suppose that $A$ is a non-cyclic word. If $B=C A D$ or $B=(C A D)$ for some $C$ and $D$, then we say that $A$ is a subword of $B$. In the case where both $C$ and $D$ are empty we consider $A$ to be a proper subword of $(A)$. Obviously, the relation "to be a subword of" is a partial ordering on an arbitrary set of 
Words. Note that each cyclic word is a maximal element in this ordering. If $A=a_{1} \ldots a_{r}$ is a word, we write $\circ A$ for $a_{1}$ and $A$ ofor $a_{r}$. Note that the symbols $\circ A$ and $A$ 。 are defined for non-cyclic words only.

2. Realizability. An admissible set $W=\left\{W_{1}, \ldots, W_{k}\right\}$ of walks in a graph $G$ is said to be realizable if there is an embedding $i: G \subset X$ of $G$ on an oriented surface $X$ with rotation $P$ such that $W_{1}, \ldots, W_{k}$ appear as cyclic factors of the corotation $P \omega$. In this case we say that $i$ is a relative embedding of $G$ with respect to the set $W$ and write $i:(G, W) \subset X$. The faces of the embedding $i$ whose boundaries belong to $W$ are inner and the remaining ones are outer. Any embedding of $G$ can be considered as a relative embedding with $W=\emptyset$; in this case each face of the embedding is an outer face. Thus we regard $\phi$ as a realizable set.

Let $G$ be a graph and $W$ an admissible set of walks in $G$. Define $K(G, W)$ to be a 2-complex obtained from $G$ by attaching a 2-cell to every closed walk $W_{i} \in W$. Each 2-cell is given an orientation consistent with that of the corresponding $W_{i}$. Note that the cell complex $K(G, W)$ need not be pure. At the same time, the admissibility of $W$ ensures that $K(G, W)$ is a subspace of some generalized pseudosurface. It is easy to see that the set $W$ is realizable if and only if $K(G, W)$ is a subspace of an oriented closed surface.

In what follows we show how to decide realizability in a simple algebraic manner. In order to do this we associate with each vertex $v$ of $G$ a set $M(v)$ of words over the alphabet $D(v)$ as follows. Let $M(v)$ be the set of all words $A$ over $D(v)$ which are maximal with respect to the following property: $a b$ is a subword of $A$ if and only if $W\left(a^{-1}\right)=b$ (recall that $W$ is a permutation!). Topologically, the words in $M(v)$ correspond to the connected components of sufficiently small deleted neighbourhoods of $v$ in $K(G, W)$. Observe that each symbol of $D(v)$ is contained in exactly one word of $M(v)$. Note that for $W=\varnothing$ each word in $M(v)$ has length 1 .

The above geometrical observation on realizability can now be formulated as follows:

Proposition 1. Let $G$ be a connected graph and $W$ an admissible set of walks in $G$. Then $W$ is not realizable if and only if there is a vertex $v$ in $G$ such that $M(v)$ contains a cyclic word of length less than $\operatorname{deg} v$.

Proof. Obviously, if some $M(v)$ contains a cyclic word of length less than $\operatorname{deg} v$, then $K(G, W)$ cannot be a subspace of any closed surface.

Conversely, let $M(v)$ contain no cyclic word of length less than $\operatorname{deg} v$ for each $v$ in $G$. Then any cyclic permutation $\bar{Q}_{v}$ of $M(v)$ determines a cyclic permutation $Q_{v}$ of $D(v)$, that is, a local rotation at $v$. It is a matter of routine to check that the product

$$
Q=\prod_{v} Q_{v}
$$

determines a relative embedding of $G$ with respect to $W$. 
Let $G$ be a graph and $W$ a realizable set of walks in $G$. Define a local relative rotation with respect to $W$ at $v$ as a cyclic permutation of words in $M(v)$. A relative rotation of $G$ with respect to $W$ is then the product of local relative rotations over all vertices of $G$.

Let $i:(G, W) \triangle X$ be a relative embedding with rotation $P$. This embedding induces a relative rotation with respect to $W$ in the following manner. For each vertex $v$ define a permutation $\bar{P}_{v}$ of $M(v)$ by

$$
\bar{P}_{v}(A)=B \text { if and only if } P_{v}\left(A_{\circ}\right)={ }_{\circ} B
$$

when $M(v)$ does not consist of a cyclic word, and

$$
\bar{P}_{v}=\text { identity }
$$

otherwise. Thus, $\circ \vec{P}_{v}(A)=P_{v}(A \circ)$ for a non-cyclic $A$. It can be easily seen that this definition is correct. The desired relative rotation is then simply the product

$$
\bar{P}=\prod_{v} \bar{P}_{v} .
$$

Note that if $W=\emptyset$, then $\bar{P}=P$.

Conversely, if $W$ is realizable, then any relative rotation with respect to $W$ induces a relative embedding of the pair $(G, W)$, as described in the second part of the proof of Proposition 1. It is clear that the permutations $P$ and $\bar{P}$ induce each other. This fact readily implies an extended form of a theorem usually attributed to Edmonds.

Proposition 2. Let $G$ be a connected graph and $W$ a realizable set of walks in $G$. The oriented relative 2-cell embeddings of $G$ with respect to $W$ are in a 1-1 correspondence with the relative rotations of $G$.

3. Interpolation property. Ordinary 2-cell embeddings of graphs are known to have the interpolation property in the following sense: if a connected graph $G$ has 2-cell embeddings on surfaces of genera $g$ and $g^{\prime}$, then it has a 2-cell embedding on a surface of genus $h$ for every $h$ such that $\dot{g} \leqslant h \leqslant g^{\prime}$. This is a classical result of Duke [1]. As we shall see below, a similar property remains valid for relative embeddings.

One way to prove this result is to reduce the problem to standard techniques. We prefer here another approach which consists in direct manipulating with relative rotations, preserving the way of thinking as in [9].

Given a rotation $P$ of $G$, let $S_{v}$ be a permutation of $D(v)$ defined by

$$
S_{v}(a)=\omega(P \omega)^{m(a)}(a) .
$$

where $n(a)$ is the least positive integer such that $(P \omega)^{n(a)+1}(a)$ is also in $D(v)$. To compute the image of an arc $a$ in $S_{v}$ one has to proceed along the oriented boundary of the face containing the arc $a$ until it enters the vertex $v$, 
say, by an arc $b$. Then $S_{v}(a)=b^{-1}$. The way $S_{v}(a)$ can be computed suggests the term local circulation at $v$ as an appropriate name for the permutation $S_{v}$. The product

$$
S=\prod_{v} S_{v}
$$

over all vertices of $G$ is a permutation of $D(G)$ and is called a circulation of $G$; obviously, $S$ depends on $P$. Its usefulness resides in the fact that the number of faces incident with an arbitrary vertex $v$ in the embedding given by a rotation $P$ can be expressed simply as $\left|P_{v} S_{v}\right|$, the number of cycles of the composition of $P_{v}$ and $S_{v}$ (see [9]). Our aim is to obtain an analogous formula for the number of outer faces at a specified vertex for relative embeddings.

Let $G$ be a graph and $W$ a realizable set of walks in $G$. Assume that a relative embedding of the pair $(G, W)$ is given via the rotation $P$. Let $S$ be the circulation induced by $P$. The local relative circulation $\bar{S}_{v}$ is a permutation of $M(v)$ defined as follows:

$$
\bar{S}_{v}(A)=B \text { if and only if } S_{v}(\circ A)=B \text { 。 }
$$

when $M(v)$ does not consist of a cyclic word, and

$$
\bar{S}_{v}=\text { identity }
$$

otherwise. Thus, $\bar{S}_{v}(A) \circ=S_{v}(\circ A)$. The definition is meaningful even in the case where $M(v)$ consists of a single non-cyclic word.

Put

$$
\bar{S}=\prod_{v} \bar{S}_{v}
$$

This permutation is a relative circulation of $G$ with respect to $W$.

Note that, similarly as for $P$ and $\bar{P}$ above, the permutations $S$ and $\bar{S}$ induce each other. Again, if $W=\emptyset$, then $S \stackrel{\varphi}{=} \bar{S}$.

For a more convenient formulation of the next result, one more notation appears to be useful. Denote by $V_{0}$ the subset of all vertices $v$ of $G$ for which $M(v)$ does not consist of a single cyclic word.

Now we are ready to formulate and prove a generalization of Stahl's counting theorem ([9], Theorem 2).

Proposition 3. Let $i:(G, W) \subset X$ be an embedding with the relative rotation $\bar{P}$ and let $\bar{S}$ be the corresponding relative circulation. Then the number 'of outer faces of the embedding $i$ incident with $v \in V_{0}$ is equal to $\left|\bar{P}_{v} \bar{S}_{v}\right|$.

For $v \notin V_{0}$ the number of outer faces incident with $v$ is, obviously, zero.

Proof. First, let us express the corotation $P \omega$ in the form $P \omega=Q W$. Thus, $Q$ is a product of boundaries of the outer faces. Now, let us examine which symbols of $D(G)$ appear in $Q$. Clearly, $a \in D(G)$ is in a cycle of $Q$ if and 
only if its initial vertex $v$ belongs to $V_{0}$ and there is some $A \in M(v)$ such that $a=\circ A$. In other words, $Q$ contains only the initial symbols of maximal words. In what follows, the set created by these symbols will be denoted by $I$.

Further, we shall pursue how the permutation $P_{v} S_{v}$ acts on $I$. Choose a vertex $v \in V_{0}$ and take $A \in M(v)$. Then

$$
P_{v} S_{v}(\circ A)=P_{v}\left(\bar{S}_{v}(A) \circ\right)=\circ \bar{P}_{v} \overline{S_{v}}(A) .
$$

That is, for $A, B \in M(v)$ we have $P_{v} S_{v}(\circ A)={ }_{\circ} B$ if and only if $\bar{P}_{v} \bar{S}_{v}(A)=B$. It follows that $P_{v} S_{v}$ restricted to $I$ is a permutation on $I$. Moreover, the cycles of $\bar{P}_{v} \bar{S}_{v}$ and those of $P_{v} S_{v}$ restricted to $I$ are in a 1-1 correspondence.

Now, assume that cycle $D=(a A b B c C \ldots Z)$ is in $Q$, i.e., $D$ is the boundary of an outer face of the embedding $i$ incident with $v \in V_{0}$. Then $(a b c \ldots)$ is a cycle of $P_{v} S_{v}$ consisting only of the arcs from $I$. However, according to Stahl's counting theorem [9], the number $\left|P_{v} S_{v}\right|$ is equal to the number of faces incident with $v$. In view of the correspondence between the cycles of $P_{v} S_{v}$ restricted to $I$ and the cycles of $\bar{P}_{v} \bar{S}_{v}$ we conclude that $\left|\bar{P}_{v} \bar{S}_{v}\right|$ is the number of outer faces incident with $v$.

Now we turn to the study of some modifications of local relative rotations. Proposition 3 allows us to replace computations with rotations by computations with relative rotations. This possibility leads directly to the following result whose proof is analogous to that of Stahl ([9], Theorem 4).

Proposition 4. Let $i:(G, W) \subset X$ be a relative embedding with a relative rotation $\bar{P}$. Let $\bar{P}^{\prime}$ be a relative rotation of $(G, W)$ obtained by interchanging in $\bar{P}_{v}\left(v \in V_{0}\right)$ two maximal words $A, B \in M(v)$, that is,

$$
\bar{P}_{v}^{\prime}=(A B) \bar{P}_{v}(A B)
$$

Let $j:(G, W) \hookrightarrow Y$ be a relative embedding given by $\bar{P}^{\prime}$. Then the numbers of outer faces in $i$ and $j$ differ by $-2,0$ or 2 .

Since the relative rotation associated with a given relative embedding of the pair $(G, W)$ can be transformed into the relative rotation associated with any other relative embedding of $(G, W)$ by a finite sequence of alternations of the same type as in Proposition 4, we have

THEOREM 1 (interpolation theorem for relative embeddings). Let $G$ be a connected graph and $W$ a realizable set of walks in $G$. Assume that the pair $(G, W)$ has a relative 2-cell embedding on surfaces of genera $g$ and $g^{\prime}, g \leqslant g^{\prime}$. Then $(G, W)$ has a relative 2-cell embedding on a surface of genus $h$ for every $h$ such that $g \leqslant h \leqslant g^{\prime}$.

4. Miscellanea. For an arbitrary connected graph $G$ and a realizable set of walks $W$ we define the relative genus $\gamma(G, W)$ of $G$ with respect to $W$ (or the genus of the pair $(G, W)$ ) as the minimum genus of the surface on which 
$G$ has a relative 2-cell embedding with respect to $W$. By analogy one can define also the maximum relative genus $\gamma_{M}(G, W)$. Theorem 1 then asserts that genera of surfaces on which $G$ has a relative 2-cell embedding with respect to $W$ fill up the whole interval of integers between $\gamma(G, W)$ and $\gamma_{M}(G, W)$

It is possible to find examples which show that genus and relative genus are sufficiently independent parameters. The same is true for the maximum genus.

It follows from Theorem 2 that if $|M(v)| \leqslant 2$ for each vertex $v$ of $G$, then surely $\gamma(G, W)=\gamma_{M}(G, W)$. In this case there is, in fact, a unique embedding of the pair $(G, W)$.

As an example, let us consider a cubic Hamiltonian graph $G$ with the set $W$ containing a single Hamiltonian circuit $H$ in $G$. In this simple case it is possible to determine exactly the value of $\gamma(G, H)=\gamma_{M}(G, H)$. As shown in [2] and [7] this number appears to be useful even outside graph theory. In both cases, $\gamma(G, H)$ was expressed as the one-half-rank of a certain $(0,1)$ matrix. We show how $\gamma(G, H)$ can be computed in a different but simple manner using permutations only. Let $\alpha_{1}$ be the cyclic permutation of vertices of $G$ induced by the orientation of $H$. Each chord of $H$ can be regarded as a transposition of its two end vertices. Let $\alpha_{2}$ be the product of such transpositions over all the chords of $H$. Note that this product is at the same time the decomposition of $\alpha_{2}$ into cyclic factors.

Proposition 5. In the above notation, the number of outer faces of the unique relative 2-cell embedding of a cubic Hamiltonian graph $G$ with respect to a Hamiltonian circuit $H$ is equal to $\left|\alpha_{1} \alpha_{2}\right|$. Then

$$
\gamma(G, H)=\frac{1}{2}\left(1+n-\left|\alpha_{1} \alpha_{2}\right|\right)=\frac{1}{2}\left(\left|\alpha_{1}\right|+\left|\alpha_{2}\right|-\left|\alpha_{1} \alpha_{2}\right|\right) .
$$

Proof. Let $P \omega=R \cdot H$, where $R$ is the product of cycles corresponding to outer faces of the relative embedding of $(G, H)$ whose rotation is $P$. Observe that each cycle $C$ of $R$ has even length since in $C$ a chordal arc $e$ is followed by an $\operatorname{arc} e^{\prime}$ on $H$ but with the orientation opposite to that of $H$, and conversely. Let $e$ and $f$ be two chordal arcs at $u$ and $v$, respectively. To complete the proof it suffices to show that $e e^{\prime} \mathrm{ff}^{\prime}$ is a subword of a cycle in $R$ if and only if $\alpha_{1} \alpha_{2}(u)=v$. However, this is an easy task and we leave it to the reader.

Another class of graphs with easily computable relative genus is that of 4-regular graphs with respect to oriented 2-factorizations. Let $G$ be a connected 4-regular graph. By an oriented 2-factorization $F$ of $G$ we understand a 2-factorization in which each circuit has a definite orientation. This enables us to consider each oriented circuit of $F$ as a cyclic permutation of its edges. Let $W$ be the collection of all oriented circuits of $F$. According to Proposition 1, $W$ is always realizable: For the sake of convenience we speak simply of relative embeddings with respect to the oriented 2 -factorization $F$ 
rather than with respect to $W$. Note that, again, the embedding of the pair $(G, F)$ is uniquely determined and, consequently, $\gamma(G, F)=\gamma_{M}(G, F)$.

Let $F=\left\{F_{1}, F_{2}\right\}$ be an oriented 2-factorization of $G$. Each oriented circuit of $F_{i}$ induces a cyclic permutation of its vertices. Let $\alpha_{i}$ be the permutation of vertices of $G$ obtained as the product of all cyclic permutations corresponding to oriented circuits in $F_{i}(i=1,2)$.

Similarly as above, it can be shown that a formula analogous to that for cubic Hamiltonian graphs holds also in this case.

Proposition 6. In the above notation, the number of outer faces of the unique relative embedding of a connected 4-regular graph $G$ of order $p$ with respect to an oriented 2-factorization $F$ is equal to $\left|\alpha_{1} \alpha_{2}\right|$. Then

$$
\gamma(G, F)=1+\frac{1}{2}\left(p-\left|\alpha_{1}\right|-\left|\alpha_{2}\right|-\left|\alpha_{1} \alpha_{2}\right|\right) .
$$

It turns out that the formulas in Propositions 6 and 7 express the relative genus by means of what is known as the genus of a pair of permutations $\alpha_{1}$ and $\alpha_{2}$. This notion was introduced by Jacques [4] and interpreted topologically by Stahl [10].

Let us finish with an example where the relative embedding is not unique: the complete graph $K_{n}$ with respect to a specified circuit $C$. To establish a formula for $\gamma_{M}\left(K_{n}, C\right)$, the edge adding technique of [5] may be used. However, instead of taking a splitting tree (see [5] for the definition) as a starting graph for this procedure, one has to take a suitable connected unicyclic spanning subgraph containing $C$. We then have Then

Proposition 7. Let $C$ be a circuit of the complete graph $K_{n}$ of order $n$.

$$
\gamma_{M}\left(K_{n}, C\right)=\left\lfloor\frac{1}{4} n(n-3)\right\rfloor .
$$

In particular, $\gamma_{M}\left(K_{n}, C\right)$ is independent of the length of $C$.

\section{References}

[1] R. A. Duke, The genus, regional number, and Betti number of a graph, Canad. J. Math. 18 (1966), pp. 817-822.

[2] R. Z. Goldstein and E. C. Turner, Applications of topological graph theory to group. theory, Math. Z. $165^{\circ}(1979)$, pp. 1-10.

[3] N. P. Homenko, The method of $\varphi$-transformations and some of its applications (in Ukrainian, English summary), pp. 35-94 in: $\varphi$-Transformations of Graphs, Math. Inst. of Ukrainian Acad. Sci., Kiev 1973.

[4] A. Jacques, Sur le genre dune paire de substitutions, C. R. Acad. Sci. Paris Sér. A 267 (1968), pp. 625-627.

[5] M. Jungerman, A characterization of upper-embeddable graphs, Trans. Amer. Math. Soc. 241 (1978), pp. 401-406.

[6] M. L. Marx, Graph imbeddings and branched extension of curves in oriented surfaces, Congr. Numer. 33 (1981), pp. 205-212. 
[7] V. P. Nekrasov, On planar graphs having an external access (in Russian), pp. 34-44 in: Combinatorial Properties of Convex Sets and Graphs, Acad. Sci. of USSR, Ural Scientific Centre, Sverdlovsk 1983.

[8] L. Oubina and R. Zuchello, A generalization of outerplanar graphs, Discrete Math. 51 (1984), pp. 243-249.

[9] S. St ahl, A counting theorem for topological graph theory, Lecture Notes in Math. 642, pp. 535-544, Springer, Berlin 1976.

[10] - A combinatorial analog of the Jordan curve theorem, J. Combinatorial Theory (B) 35 (1983), pp. 28-38.

[11] K.-P. Vo, Segment graphs, depth-first cycle bases, 3-connectivity, and planarity of graphs, Linear and Multilinear Algebra 13 (1983), pp. 119-141.

[12] A. T. White, Graphs, Groups and Surfaces, 2nd edition, North-Holland, Amsterdam 1984.

FACULTY OF MATHEMATICS AND PHYSICS

COMENIUS UNIVERSITY

842 IS BRATISLAVA

CZECHOSLOVAKIA
DEPARTMENT OF MATHEMATICS FACULTY OF CIVIL ENGINEERING SLOVAK TECHNICAL UNIVERSITY 81368 BRATISLAVA CZECHOSLOVAKIA 